\title{
Artificial Intelligence for Smart Systems Critical Analysis of the Human Centered Approach
}

\author{
${ }^{1}$ Zoran Galic Hajnal \\ ${ }^{1}$ Faculty of Science, University of Novi Sad, Trg Dositeja Obradovića 6, Novi Sad 106314, Serbia \\ 'hajnalgalic543@aol.com
}

\author{
Article Info \\ Journal of Computing and Natural Science (http://anapub.co.ke/journals/jens/jens.html) \\ Doi: https://doi.org/10.53759/181X/JCNS202101013 \\ Received 25 December 2020; Revised form 30 January 2021; Accepted 25 March 2021. \\ Available online 05 July 2021. \\ (C2021 Published by AnaPub Publications.
}

\begin{abstract}
A program for Artificial Intelligence (AI) is knowledge as intelligent agent, which typically interacts with the ecosystem. This agent is capable of identifying the status of the ecosystem using the sensors before affecting the state via the actuators. We call the smart systems "agents" whenever they are able to make some decisions on their own with respect on particular goals. On the other hand, Machine Learning (ML) signifies a specific strategy meant to design smart systems whereby these systems can adapt to specific behaviors with respect to data. In the modern age, humans are rapidly collaborating with ML and $\mathrm{AI}$ systems. The AI that is human-based is a perspective of ML and AI, which algorithms have to be established with the awareness that they are a major segment of the massive system incorporating human. In this paper, we have presented a research that means that AI systems understand humans with respect to their socio-cultural aspects and that AI system assist humans comprehend them. We also present an argument of the challenges of social responsibility e.g. transparency, interpretability, accountability and fairness.
\end{abstract}

Keywords - Artificial Intelligence (AI), Machine Learning (ML), Human-Centered Artificial Intelligence (HCAI)

\section{INTRODUCTION}

The concept of Artificial Intelligence (AI) signifies the research and establishment of algorithms, which are capable of performing tasks or action, which people deem to necessitate the intelligence of humans. Construed widely, a smart system can assume different forms: a system established to be indistinguishable from people; speech assistance e.g. Google Assistance, Cortana, Siri, or Alexa; self-driving vehicle; an e-commerce site with a recommender; or a nonplayer element within video games. Whenever a smart system is able to make a number of decisions on its own in relation to specific goals, we call it an agent.Machine Learning (ML), on the other hand, is a certain methodology meant to formulate smart systems in which the system is capable of adapting it behaviours based on the provided data. ML algorithms, in particular, are responsible for the recent growth of AI commercialization, as evidenced by this award. People are progressively contacting ML and AI systems. In various times, it is evident, just like in speech recognition systems, such as Google Assistance, Cortana, Alexa, and Siri. Autonomous vehicles and computer games with non-player characters are examples of this. When it comes to algorithms working behind the scenes to project certain services or products or even used for loan approval, the proof is unclear. Due to the fact that intelligent systems have the capacity to affect the living standards of humans, it is fundamental to establish them within this mindset.

Algorithmic developments in machine learning and artificial intelligence (AI) alone do not suffice for interacting with or around people [1]; this is widely acknowledged. The Human-Centered Artificial Intelligence (HCAI) signifies a perspective on ML and AI, which smart systems have to be established based on the awareness that they are a major segment of the larger systems integrating human stakeholders e.g. clients, operators, users and other individuals in the neighbourhood. Some AI practitioners and researchers have begun to utilize the terminology "Human-Centred Artificial Intelligence (HCAI)" to signify smart systems, which are established with social responsibility to focus on challenges such as transparency, interpretability, accountability and fairness. These are the fundamental challenges.

HCAI encompasses more than the challenges presented above and this desiderates we investigate the wide-rage scope of what is signifies to have AI that is human-based, integrating the factors, which underlie our present needs for transparency, interpretability and fairness. At the basis of HCAI is the recognition that the manner smart systems mitigate issues, mostly when utilizing ML, is critically "alien" to people without training in AI or computer sciences. We have a tendency of interacting with other individuals, and we have formulated a more dynamic capacity to forecast what other individuals will do and why. This is mostly known as the "theory of mindset" and this makes it possible to hypothesize the intentions, goals, beliefs, desires, and actions of others. However, our "theory of mindset" 
typically breaks down whenever interacting with the smart systems that do not mitigate the challenges accordingly and can come up with unexpected or unusual remedies even when operating as projected. In cases where the smart systems are black boxes, the problem is even worse.

In machine learning and artificial intelligence, the term "black box" refers to situations in which users are unable to determine the algorithms being used, or in which the systems are so complex as to defy simple inspection. Irrespective of whether the smart systems are black boxes or not, there are evident interactions between the smart systems and the individuals that are not professionals in computer science or AI [2]. It is fundamental to firstly comprehend the manner, in which we can establish smart systems that are able to assist people to comprehend their decision. Secondly, the things humans need to do concerning the smart systems to be trustworthy when making decisions or be comfortable when working with the smart systems. Thirdly, the possibilities of smart systems conveying this data to the users in a comprehensive and meaningful way, is another key issue to consider.

HCAI acknowledges that humans can be as incomprehensible as intelligent systems. The speech processing and language aspects of smart systems' ability to understand humans are usually the focus of our attention. Activity recognition, speech processing and natural language are significantly concerns in developing fundamental and interactive smart systems. For the purpose of effectiveness, ML and AI systems require a "theory of mindset" concerning humans [3]. We use common sense comprehension to predict and interpret the actions of other people in much the same way. Our socio-cultural perspective has a profound impact on who we are as individuals.

Smart systems, on the other hand, do not develop as rapidly in a culture and society as humans do. People inhabit an ecosystem that is defined by sociocultural norms and beliefs. A smart system that can model the sociocultural norms and beliefs might be capable of disambiguating the behaviours of humans and make more trained projections regarding how to project and respond to the needs of humans. More intelligent systems, which comprehend the sociocultural underpinning of the people, might be in minimal chances of committing errors for issues that people do not take serious, rendering them safer for use, safer to bring closer to people, at least on a minimum level. In the future, it's possible that smart systems will be able to evaluate their own behavior for consistency with ethical behavior in terms of fairness.

Human-based machine learning (ML) and artificial intelligence (AI) systems are presented in this paper with a major basis in understanding socio-cultural norms and being able to produce details that non-professional end-users can understand. The paper concludes with some suggestions for future research and development. It is from these capacities that most of our AI needs are derived, and they contribute to our social advantage. To achieve this rationale, the sections below have been organized as follows: Section II focuses on the literature review of the topic. Section III critically evaluates the paper with major focus on Overview of Artificial Intelligence (AI); Comprehending Humans; AI Systems Assisting Humans for Comprehension; The Requirements for Human-Centred Artificial Intelligence (HCAI); and Efforts of Firms to Establish AI Design Guidelines. Finally, Section IV concludes and provides future directions regarding the research.

\section{LITERATURE REVIEW}

C. Weisbin in [4] explain the significant aspect of AI in controlling policies of agents. From the analysis, it is viewed that the control policies of agents imply the manner in which inputs are obtained from the sensors and how they can be translated to the actuators. In other words, the research explains how the sensors can be mapped to the actuators, and this is made possible using the functions available within the agents. The fundament obligation of Artificial Intelligence (AI) is to structure human-like intelligence within machines. Nonetheless, this dream can be fulfilled via the learning algorithms that focus on mimicking the manner in which the human mind learns things.

D. Shubham, P. Mithil, M. Shobharani and S. Sumathy in [5] evaluated the aspect of Machine Learning (ML) that is a segment that had developed from AI. Based on the research, it is of much significance since it allows machines to gain human-like intelligence without integrating programming that is explicit. Nonetheless, the AI programs do the significantly interesting things like photo tagging, web search or email anti-spam. In that case, ML was established as a novel capacity for computers and in the modern age, it focuses on the various fields of industries and basic sciences. There are autonomous robotic systems, and computing biology in the present age. The research posits that approximately $90 \%$ of data across the globe was produced in the last two years and the incorporation of ML library identified as Mahout into the systems of Hadoop ecosystem has allowed us to face the issues of Massive Data, mostly the unstructured data.

P. Pereira in [6] argue that in the field of ML research, the major focus is dedicated to developing and choosing algorithms and conducting the experiments based on the available algorithms. These significantly biased perspectives minimize the implication or real-life applications. In this analysis, different application under appropriate group of ML has been presented. This research focuses on bringing the major segments of application under a single umbrella and presents a generalized and realistic perspective of the real-life application. Aside from these two application recommendation have been put forward. The segment of ML is so wide and rapidly growing that it possibly proves to be fundamental in automating different life facets.

J. Mei in [7] also conducted a research on ML and define it as a segment of research providing computers with the capacity to learn without being programmed explicitly. The researchers were known globally for their checkers playing programs. Initially, when they developed the checkers playing programs, they were better compared to the programs. However, as time went, these checkers playing programs learned what effective board positions were, including the bad positions when playing various games. 
P. Jauffret, T. Hanser, C. Tonnelier and G. Kaufmann in [8] provided a formalized definition of ML as a computer program that learns from Performance measures $(\mathrm{P})$, Tasks $(\mathrm{T})$ and Experience $(\mathrm{E})$. In case it is measured by $\mathrm{P}$ and its performance on $\mathrm{T}$ enhances with the experience $\mathrm{E}$ then this program is identified as the ML program. As for the checkers playing sample, the experience $\mathrm{E}$ was the experience of considered the program play certain gains against itself. The task T represents the tasks of checkers playing. A performance measure P represents the probability that is wins the upcoming games of the checkers against a number of novel opponents. It is argued that in the various engineering fields, there are larger sets of data, which are being comprehended using algorithmic learning.

Researchers in [9] defined the concept of Artificial Intelligence (AI) as a technology that is able to behave based on human-like intelligence. With the advancements made over the past few decades, AI has become considerably pervasive. Many insurance firms utilize Ai in the processing of claims and financial institutions depend on the automated stock trading. People are capable of performing individual checks for skin cancer, utilizing the smart apps e.g. Health AI- skin Cancer or SkinVision or they can potentially make contact with the smart services via the user interfaces e.g. Amazon Echo or Google Home, that are considered to be intelligence since they are capable of comprehending the natural language questions and provide the feedback using the same natural language.

D. Petrelli, A. Dadzie and V. Lanfranchi in [10] posit that many users of the AI technology do not have enough insights into the interior operations to comprehend the manner in which they have reached their output. As a result, this makes it challenging for humans to trust in advancements, learn from it and be capable of correcting the predictions of the upcoming situations. One of the considerable samples of the application of AI is Google's search engine. Many users of the web neither understand or known the algorithms that the search engine uses to find the respective search outputs that typically match what they inquired. One effect is that the owners of the website who expect their users to find their webpages must employ the Search Engine Optimization (SEO) professionals to manipulate the content of their webpages and format it in means, which enhance the rankings of their web pages. The SEO professionals do not have full comprehension of the algorithms of Google either, but they are familiar with the transitions make to webpages to have a significant implication on the rankings.

O. Bihun and M. Miłosz in [11] describe Google's search algorithms as a significant sample of the paradigm known as the black-box. Nonetheless, comparing Ai to the black box is not enough to describe it effectively since the black box itself is considered predictable based on outputs to inputs. In Ai, due to the condition of self-learning algorithms, the usage of technologies might not be the case. For instance, the Nest Smart Thermostat selects temperature settings, which are incomprehensively for various users. In reference to the researchers, it neither gave an explanation of its decision-making nor permitted users to make an override of the settings.

\section{CRITICAL ANALYSIS}

\section{Overview of Artificial Intelligence (AI)}

The terminology "Artificial Intelligence (AI)" was initially utilized by John McCarthy in the 1956 conference held at Dartmouth. From that time, the concept of AI has undergone three significant booms during the times of technological and scientific development. The $1^{\text {st }}$ boom was dated from 1956 - 1976. From the late 1950s, people had effectively introduced the first ideology of the Chat software and the Neural Network (NN) software and provided a proof of the fundamental mathematical theories that exclaimed that the era of AI is gradually approaching and that the robot systems will exceed the intelligence of humans in the next ten years. In the $2^{\text {nd }}$ boom i.e. from 1976 to 2006 , big data and Hopfield Neural Networks learning algorithms were proposed in 1980s and these technologies mad AI significantly popular and initiated the introduction of speech recognition, speech translation planning and the $5^{\text {th }}$ Generation $(5 \mathrm{G})$ computer perspective. Nonetheless, these perspectives fell through and the $2^{\text {nd }}$ boom was introduced again. Following data accumulation to a considerable amount, some of the results would have halted to a significant extent. In the $3^{\text {rd }}$ boom i.e. from 2006 to the present, AI was evident once again as deep learning was presented in 2006 [12]. In addition, ImageNet rivalry was evidence in the aspect of image identification in 2012.

Presently, humans have embraced the significant development in different fields e.g. brain theories, brain science, quantum physics, neuroscience, and cognitive psychology that relate to AI have continued to advance. Without the incorporation of the developments in computer science with linguistics, neuropsychology, brain science and other fundamental disciplines, the Research and Development (R\&D) efforts on AI would not have amounted to fundamental results. The research analysis on AI has also led to fundamental research highlights e.g. ML, NN, Genetic Algorithm (GA), Particle Swarm Optimization (PSO), Support Vector Machine (SVM), and Expert System (ES). AI has widely been applied in every aspect of human living, even exceeding the intelligence of humans to complete recognition tasks, decision controls and decision-making. As for recognition, AI can differentiate, categorize, and retrieve data. In the aspect of the process of making proper decisions, AI is capable of carrying out numerical object assessment and matching. Concerning the control, AI is capable of completing the performance generation, designing and optimization of actions, and automation of operations.

Nonetheless, social, technological and scientific issues arisen from the enhancement of AI are attracting more attention from the general public. Whereas promoting social development, the widespread AI application has various prominent negative effects. For instance, the applications of robotic systems have amounted to increased degree of unemployment; application of AI has broadened the gap of wealth; the algorithms of AI have led to bias; massive data has amounted to the leakage of privacy and degeneration of the spiritual life for humans. All these are considered social issues stimulated by the application of AI developing. P. Marx in [13] presented an analysis of the employment 
risks of mental and manual labour influenced by AI. For manual employers, structured tasks with weaker social interactions and lower skillset are facing more risks, e.g. truck drivers, sewing employees, and fast food cooks. For the mental employees, those involved in weaker social and lower creative tasks are in significant unemployment danger, e.g. telemarketers and radiologists.

In the modern age of AI, it is unavoidable for some jobs or occupations to be replaced. In case your jobs If your tasks does not necessitate much skills and talents can be attained through learning, if what you have to do is a lot of repetitive task without critical thinking, and if you operate in narrower social networks and have minimal communication or contact with others, then you are possibly likely to be substituted by AI. The reconstruction and deconstruction of the occupational system is just a single aspect of what AI has presented. Various individuals even put forward the "machine risk theory." Comprehending AI is considered the prerequisite of putting it into application. AI represents the simulation of human intelligence including its developments based on the algorithmic breakthroughs.

Considering this connection and human's concerns and confusion in comprehending AI, this paper first evaluates the condition of AI, and consciousness, including the characteristics and merits of cognitive computing, and project the upcoming generation of AI development. The subsection below evaluates the comprehension of humans following the development of AI.

\section{Comprehending Humans}

Various AI systems, which interact with the humans, will have to comprehend the manner in which humans act and what they expect. This will make them significantly applicable and also safe to utilize. There are approximately two approaches in which comprehending humans can be beneficial to smart systems. Firstly, the smart systems have to infer what humans want. AI systems will be created in the future that receives their protocols and goals from people. In spite of this, human beings do not typically mention what they imply. Miscomprehending an individual's intention can amount to potentially perceived failure. Secondly, deciding to misunderstand written languages or human speech, consider the ideology that perfectly comprehends protocols and objectives is implicit or unstated [16].

Common sense failure objectives are considered when a smart agent does not accomplish the required results since part of the objective, or the manner in which the objective should be attained, is considered unstated (this is known as corrupted objective or the reward that is corrupted). Why could this take place? One of the reasons is that people are used to communicate with others who share knowledge concerning the manner in which the globe operates and how things are done. To ignore the fact that computers are not accustomed to sharing knowledge sharing and might take particular specifications factually is much easier than to recognize this. AI systems have failed, but human operators have failed. In autonomous agents and robotics, it is less important to structure common sense mistakes. As an example, let's say you want robots to access the chemistry and choose a drug for you. Due to their illness, they may wish for the robot to return immediately. By going to the chemistry, over the counter, selecting drugs and going back home, the robots have minimized and successfully executed resources and time. Not to mention that it "robbed" the pharmacy by not fully participating in social constructs of exchanging resources for goods [17].

One of the remedies to avoid common sense objective failure is for the smart systems to possess common sense skillset. This might be any skills commonly shared by humans from a similar community and cultural background. For example, driving on the right side of the road is a declarative skill (such as a waiter in a hotel will not present bills until they are requested to do so). While there have been various attempts to create knowledge based on common sense skill declarations, the efforts can be viewed as incomplete, and there are a dearth of information available in the process actions.

Machine visioning can be applied to cartons, videos, and images from a variety of sources. What human beings write, incorporating tales, news or encylopedias, reveals a variety of common sense skillsets. A lot of common knowledge can come from writings and stories; human being write what they are aware of, social or cultural views could originate from different illustration of the process actions e.g. attending a wedding ceremony to significantly implicit statements about what is right or wrong. A smart system's procedural skillset, in particular, can be used to effectively serve individuals by delaying their actions and detecting and responding to anomalous behavior. Predictive text completion is essential in the same way. Predicting the daily routine in its entirety can be useful.

Integrating common sense procedural skillset with the behaviour can amount to smart agents, which are safe. To the dimension that it is not possible to enumerate the "protocols" of the community that is more compared to just "societal laws" has common sense procedural skillset can assist intelligent systems and robotic systems to follow more social conventions. Most of these conventions exist to help people avoid conflict, even if they inconvenience us. According to [14], smart autonomous agents that use written tales as training demonstrations can implicitly learn to avoid socially undesirable actions. Since stories are more often compared to positive examples of behavior, this usually occurs as a side effect of the comparison process itself

As a general rule, their systems address the problem of common sense objective failure through rewarding information from a number of standardized tale progressions in closer proximity. As a result, agents' masters do not "steal" prescription drugs, because more of the tales indicate the exchange of resources, which encourages agents to choose the actions, despite the fact that it is more prompt and inexpensive to consider the opposite of what is expected." As a result, agents must be informed of what "robbing" means and why it must be avoided. Using a common sense skillset as a theoretical "basis of mindset" whenever contacting human beings, HCAI engagements could become much more natural. 
Despite the fact that AI and ML algorithms for making decisions operate distinctively from humans' decisionmaking approaches, systems' actions are more recognizable to humans as a result of this. As a result, human contact is made in a safer way: it is capable of minimizing common sense objective failures since the agents fills in an underspecified objectives with common sense procedural facts; and agents, which act based on the humans' expectations will innately mitigates the issues and conflicts with an individual who is applying the "theory of mindset" to smart agents. The subsection below provides an analysis of AI systems assisting humans to comprehend them.

\section{AI Systems Assisting Humans for Comprehension}

Autonomous robotics or smart systems agents, invariably, are capable of making mistakes, failures; violate expectations or performing actions that confuse humans. Our natural inclination is to wonder why people do things. Humans will be required to provide intelligent, autonomous systems with objectives, but the systems will be required to select and execute the details. In particular, neural networks $(\mathrm{NN})$ are often viewed as difficult to understand, implying that it takes a lot of effort to figure out why a system's response to stimulus is taken into account. We often refer to the process of "opening black boxes" in order to gain a better understanding of how autonomous decisionmaking systems work. This work focuses primarily on the visualization representations mastered by the neural networks (such as generating pictures that activate various segments of the neural network; or by tracking the impacts on output performance that different input data have) (such as masking or removing segments of the training information to effectively visualize the manner in which performance is affected).

Machine trained models could be challenging for AI experts to comprehend, and this form of operation is stimulated majorly to AI-oriented users, with the hope of troubleshooting or optimizing troubleshooting ML infrastructures.

Nonetheless, in case we expect to accomplish the objectives of autonomous agents and the robot systems utilized by end-users and the operations surrounding humans, we have to consider non-professional human operators. Whenever it comes to the interaction of bots and automated agents, these professionals have very specific needs. The non-professional operators are likely not focussed on searching for a comprehensive inspection of the interior operations of systems, but more likely searching for a solution. A solution represents a concept, which users have to be able to seek or correct compensations for the perceive faults. As a result, smart agents acted in a way that violated users' expectations, resulting in a miscalculation.

"Solution" begins with gathering enough data so that you can choose an effective course of action. Errors in sensors, dataset bias, incomplete or incorrect framework, effector error or other causes could have caused a fault or the appearance of failures. In most instances, the data can be a solution itself, in the manner of an explanation, which assists individuals to revise theories of mind concerning the agents or the admissions of any potential faults. Details are post-hoc explanations regarding the manner in which systems come to a particular behaviour or conclusion. As an example, sensory inputs can be visualized by displaying segments of sensory inputs that are responsible for the majority of outputs or by processing natural language. There has been no in-depth study on what constitutes the best sample of the actions of ML systems from the sense of cognitive factors. One of the options for natural language explanations is the production of details concerning algorithmic procedural sensory inputs. This is unsatisfactory since the algorithms e.g. reinforcement learning and NN defy easier explanations (such as the actions was taken since various trials show that the conditions similar to these the actions have the highest probabilities of maximizing the upcoming rewards").

Taking cues from how people answer the question "why did you consider doing that?" is another key option. As a result of the ideology, people create rationales and explanations to justify various actions. A decision-making process is not fully understood by humans; we invent stories based on the information available to us about ourselves and our intentions to be informative. Therefore, others may accept the rationales knowing that they are not $100 \%$ accurate reflections of the neural and cognitive processes that produced the behaviors at the time in which they are being used. Explanation or rationale generation is therefore the obligation of formulating details comparable to what people would say in case they were emulating the behaviours that agents were performing in a similar situation.

Human-like rationales, regardless of whether they reflect the internalized procedures of black-box smart systems, have been shown to increase non-professional operative autonomous and robotic systems' trust, comfort, and familiarity. Explanations are typically produced by firstly gathering details of humans undertaking the same tasks to that of the autonomous systems. After learning how to translate internal conditions of autonomous agents into natural language details found in a corpus, $\mathrm{NN}$ is trained to potentially translate internal conditions into natural language details. If the corpus contains any culturally specific idioms, the rationales are automatically generated. Open research questions include: "When is a single black box on top of another effective at providing detail? Exactly how do inaccurate details and rationales affect the perception of trust in the operators' abilities to execute?" The rationales are only a part of the solution. You can't get them to answer specific questions because they're too focused on high-level details. The rationales might be utilized from various forms of individuals.

The Requirements for Human-Centred Artificial Intelligence (HCAI)

The Human-Centred Artificial Intelligence (HCAI) considers various critical factors, which determining how bad or good users experience is for individuals who are contacting smart systems. The producers of AI technologies require 
ensuring that users comprehend how AI operates to a particular dimension. A. Demetriou, G. Spanoudis and M. Shayer in [15] conducted an evaluation of "detailed AI." Nonetheless, this segment is only a single part of more detailed analysis known as HCAI that considers various other fundamental factors, which determine how bad or good the user experiences are for individuals who are contacting the smart systems. Trust is considered one of the fundamental factors whereby a lack of it can possibly limit the proliferation of the intelligent technology, including the potentials for lifting the living standards for various individuals that results from the superior capacity of AI to make particular decisions more effective compared to humans.

Whenever humans are a major part of an AI schemes, which ingests particular inputs, manufactures them, and provide some outputs (all the instances where systems have not attained compete automations or might not be desirable to do it), a contact between AI and people needs to be crafter carefully. Nonetheless, the connection between $\mathrm{AI}$ and individuals is only a single aspect, which has to be placed into consideration. Some educational institutions have established HCAI schools to analyse and comprehend the implications of AI, both negative and positive, not just on persons but also on governments, industries, economies and social institutions. The key question remains: "What are the main ways of designing and developing AI systems to enhance collaboration and communication to make it enjoyable, efficient or effective? How can AI schemes augment the capacities of humans instead of straight out replacing people? To allow users to trust the working of machines, how can we assist them to effectively comprehend the weaknesses and strengths of AI?

There has been significant focus on the needs that HCAI have to serve human needs and therefore place people in the middle of the whole experience. Consequent to that, humans have to remain in control even in significantly automatic cases. Human automation and control are not exclusion mutually. The human-centred perspective is a progression of the field referred to as the Human Computer Interactions (HCI) that, in 1970 and 1980 allowed the wide-range adoption of custom computers. Nonetheless, whereas the HCI society has spent more than 40 years structuring standards for the interface for the graphic users, the interface have just limited applicability and the AI system values. Actually, AI best practices might just contradict the protocols. For instance ISO $9241-110$ necessitates the conformity with the individuals' expectations, but mentioned earlier on, without effective comprehension of the AI's understanding, or conceptual framework, the mental framework of users might signify false or inaccurate expectations.

Another reason for the mismatch between the classical applicability protocols and the segment of AI is that the former do not consider that the technologies are the human-based actors who are capable to pass the Turing evaluation. The expectations of the humans contacting with a smart system whose user interfaces are an agent or bot are noticeably more for the contemporary, utilitarian software instrument. Moreover, HCI applicability protocols were formed for the interfaces of the graphic users whereas the objective of the intelligent systems is to facilitate the interaction of the human system in more physical, seamless means that mimic people to people communication. Chatbots show that, for these systems, effective conversation designs are more fundamental compared to the designs of the interfaces for the graphic users.

To structure physical AI - Human conversations more realistic, it is fundamental to consider the human conversational procedures and conventions, including the biases, which amount from distinctions in status, culture and gender. Beyond the conversational factors, we require to apply the theories and frameworks from the human training, comprehension, and decision-making to formulate of AI-based technologies, enabling system bots and agents to be efficient, trustworthy individuals of their users. The objective is to formulate robust, formative frameworks and the models, which inform reusable protocols and the checklist for designing the human-based, AIcentred, interactive schemes. Whereas there are a number of initial protocols for AI-based systems, they are just starting and more works are fundamental, as industry heads acknowledge.

However, in the past few decades, we have visualized the proliferation of the AI schemes, which mimic people, not just based on their smartness, but also in their appearances and their capacity to communicate; this strategy has been met with much criticism. Many researchers have cautioned that, formulating the technical schemes e.g. service bots to copy humans might stop the designers from taking complete advantage of novel computer characteristics, which have not human being analogue - for instance, data displays or advanced sensors. The researchers have called for the critical evaluation and comprehension of when to equip AI with the human-centred characteristics and whenever to quit doing this. We visualized the equivalent at the dawn of the Human and Machine Interaction discipline in 1950 when it was considered that "machines are better at, humans are better at " lists.

\section{Efforts of Firms to Establish AI Design Guidelines}

As different forms have operated to advance the segment of AI, various firms have structured and published their custom lists of AI design protocols, which they apply.

Global interest in AI application, incorporating imaging, is significantly high and advancing, stimulated by the presence of big data (massive datasets), substantial developments in computing energy, and novel deep learning algorithms. Aside from the advancement of the novel AI strategies per se, there are various challenges and opportunities for the imaging society, integrating the advancement of the common nomenclature, effective means to share image information and validation standards of AI programs application across various image platforms and the populations of patients. AI monitoring systems might aid radiologists to prioritize the task lists by identifying any suspicious or positive instances of the earlier reviews. AI tasks and programs can be utilized to extract the radiomic data from the pictures not discernible through visual inspections, possibly enhancing the prognostic and diagnostic 
values derived from the various image datasets. The forecasts have been structured that recommend AI will place radiologists from business. This challenge has been overrated, and it is likely that the radiologist will beneficially integrate AI approaches into different practices.

Comparing those of Google and Microsoft shows the similarities they comprehend, humans' considerations and commitments, according to Table 1 below.

Table 1: Comparison of Google's and Microsoft's AI Design ideologies

\begin{tabular}{|c|c|}
\hline Google AI ideologies & Microsoft AI ideologies \\
\hline $\begin{array}{l}\text { Be structured and be present for } \\
\text { users, which accord with these } \\
\text { ideologies } \\
\text { Be accountable to humans } \\
\text { - Integrate privacy design } \\
\text { ideologies } \\
\text { Be socially advantageous } \\
\text { - Eliminate reinforcing and } \\
\text { establishing unfair biases } \\
\text { Be constructed and tested for } \\
\text { privacy and safety }\end{array}$ & $\begin{array}{l}\text { - Accountability - humans have to be } \\
\text { accountable for the various AI schemes } \\
\text { Transparency - AI schemes have to be } \\
\text { comprehensible } \\
\text { - Inclusiveness - AI schemes have to } \\
\text { empower everybody and engage humans } \\
\text { - Security and privacy - AI schemes have } \\
\text { to be safe and embrace privacy } \\
\text { Safety and reliability - AI schemes have } \\
\text { to embrace safety and reliability } \\
\text { Fairness - All AI schemes should handle } \\
\text { humans in a fair way }\end{array}$ \\
\hline
\end{tabular}

The present limitations in the presences of the technical professionalism and even computing energy will be mitigated over time and might also be addressed through remote accessibility remedies. The success of AI in image processing will be evaluated by value formed: enhanced diagnostic certainty, prompt turnaround, effective results for patients, and effective life quality for radiologists. AI provides a novel and potential collection of strategies for evaluating image data. Radiologists will evaluate these novel routes and are a leading role in the AI applications e.g. the medical sector.

\section{CONCLUSION AND FUTURE DIRECTIONS}

In this paper, an analysis on Human-Centred Artificial Intelligence (HCAI) has been done and based on two fundamental capacities: comprehending humans; and being capable to assist humans to comprehend AI systems. There might be other fundamental capacities, which this paper does not focus on. Nevertheless, it appears that the two capacities can be used to extract a number of desirable characteristics for smart systems that interact with nonprofessional users, as well as for various systems that are designed for social responsibilities. People are becoming more aware of the need for transparency and fairness when it comes to AI schemes, for example. As a result of fairness, users must be treated equally and without much prejudice in their treatment. Presently, we spend a lot of time collecting data and constructing checks into systems that are meant to prevent systems from taking unjust actions against people. While it may not be possible for a smart system to achieve the same level of fairness as a fair system, it may be possible for it to eliminate prejudice and discrimination in situations that were not anticipated by the system's designers. In terms of transparency, end-users should have access to massive datasets and the AI system's workflows. As long as AI systems are able to assist humans in understanding their choices through details or other methods that are accessible to non-professionals, people will be more willing to use them. It's possible that focusing on the details is the first step towards a solution, as it's a crucial aspect of accountability.

Human-Centred Artificial Intelligence (HCAI) does not imply that ML or AI algorithm should think like humans or be cognitively reasonable. Nonetheless, it does identify the ideology that individuals who are non-professionals in computing science or AI fall back on the "theory of mindset" formulated to facilitate interactions with other humans and draw on socio-cultural behaviours, which have been introduced to avoid the people - people conflict. Assisting humans in understanding smart systems implies building smart systems that understand human projections and needs. A research ambition (future research directions) is presented in the analysis of HCAI, which will enhance our scientific understanding of critical ML and AI, whereas providing enough support to the smart service deployment in a simultaneous manner to interact with people.

\section{References}

[1]. D. Ciuriak, "Economics of AI/ML and Big Data in the Data-Driven Economy: Implications for Canada's Innovation Strategy", SSRN Electronic Journal, 2019. Available: 10.2139/ssrn.3362083.

[2]. M. Carabantes, "Black-box artificial intelligence: an epistemological and critical analysis", AI \& SOCIETY, vol. 35, no. 2, pp. 309-317, 2019. Available: 10.1007/s00146-019-00888-w.

[3]. P. Khargonekar and M. Dahleh, "Advancing systems and control research in the era of ML and AI", Annual Reviews in Control, vol. 45, pp. 1-4, 2018. Available: 10.1016/j.arcontrol.2018.04.001.

[4]. C. Weisbin, "Real-Time Control: A significant test of AI technologies", IEEE Expert, vol. 2, no. 4, pp. 16-17, 1987. Available: 10.1109/mex.1987.5006526 
[5]. D. Shubham, P. Mithil, M. Shobharani and S. Sumathy, "Aspect level sentiment analysis using machine learning", IOP Conference Series: Materials Science and Engineering, vol. 263, p. 042009, 2017. Available: 10.1088/1757-899x/263/4/042009.

[6]. P. Pereira, "Using ML Models to Detect Malicious Traffic: Testing ML Models", International Journal for Information Security Research, vol. 10, no. 1, pp. 906-909, 2020. Available: 10.20533/ijisr.2042.4639.2020.0104

[7]. J. Mei, "Refining humane endpoints in mouse models of disease by systematic review and machine learning-based endpoint definition", ALTEX, 2019. Available: 10.14573/altex.1812231

[8]. H. Anandakumar and K. Umamaheswari, "Supervised machine learning techniques in cognitive radio networks during cooperative spectrum handovers," Cluster Computing, vol. 20, no. 2, pp. 1505-1515, Mar. 2017.

[9]. H. Anandakumar and K. Umamaheswari, "A bio-inspired swarm intelligence technique for social aware cognitive radio handovers," Computers \& Electrical Engineering, vol. 71, pp. 925-937, Oct. 2018. doi:10.1016/j.compeleceng.2017.09.016

[10]. P. Jauffret, T. Hanser, C. Tonnelier and G. Kaufmann, "Machine learning of generic reactions: 1. Scope of the project; the GR AMS program", Tetrahedron Computer Methodology, vol. 3, no. 6, pp. 323-333, 1990. Available: 10.1016/0898-5529(90)90059-h.

[11]. "Applications of Artificial Intelligence", Artificial Intelligence, vol. 114, no. 1-2, pp. 1-2, 1999. Available: 10.1016/s00043702(99)00086-7.

[12]. D. Petrelli, A. Dadzie and V. Lanfranchi, "Mediating between AI and highly specialized users", AI Magazine, vol. 30, no. 4, p. 95, 2009. Available: 10.1609/aimag.v30i4.2263.

[13]. O. Bihun and M. Miłosz, "Comparison Google's and Yandex's search algorithms", Journal of Computer Sciences Institute, vol. 4, pp. 128-130, 2017. Available: 10.35784/jcsi.609.

[14]. B. Unhelkar and T. Gonsalves, "Enhancing Artificial Intelligence Decision Making Frameworks to Support Leadership During Business Disruptions", IT Professional, vol. 22, no. 6, pp. 59-66, 2020. Available: 10.1109/mitp.2020.3031312.

[15]. P. Marx, "Labour market risks and political preferences: The case of temporary employment", European Journal of Political Research, vol. 53, no. 1, pp. 136-159, 2013. Available: 10.1111/1475-6765.12027.

[16]. N. Shah and Y. Jani, "Implementation of Smart Infusion Pumps: A Scoping Review and Case Study Discussion of the Evidence of the Role of the Pharmacist", Pharmacy, vol. 8, no. 4, p. 239, 2020. Available: 10.3390/pharmacy8040239.

[17]. A. Demetriou, G. Spanoudis and M. Shayer, "Developing intelligence: Is a comprehensive theory possible?", Intelligence, vol. 41, no. 5, pp. 730-731, 2013. Available: 10.1016/j.intell.2013.07.017. 